\title{
Erratum to: Enhanced Extracellular Production of Heterologous Proteins in Bacillus subtilis by Deleting the C-terminal Region of the SecA Secretory Machinery
}

Hiroshi Kakeshita $\cdot$ Yasushi Kageyama •

Katsutoshi Ara $\cdot$ Katsuya Ozaki $\cdot$ Kouji Nakamura

Published online: 16 July 2010

(C) Springer Science+Business Media, LLC 2010

Erratum to: Mol Biotechnol

DOI 10.1007/s12033-010-9295-0

The surname of the first author was misspelled in the original publication. The correct spelling is Hiroshi Kakeshita.

The online version of the original article can be found under doi:10.1007/s12033-010-9295-0.

H. Kakeshita $(\bowtie) \cdot$ K. Nakamura

Graduate School of Life and Environmental Sciences, University

of Tsukuba, 1-1-1 Tennoudai, Tsukuba, Ibaraki 305-8572, Japan

e-mail: kakeshita.hiroshi@kao.co.jp

H. Kakeshita · Y. Kageyama · K. Ara · K. Ozaki

Biological Science Laboratories, Kao Corporation, 2606

Akabane, Ichikai-Machi, Haga-Gun, Tochigi 321-3497, Japan

藻 Humana Press 